ISAHP 2001, Berne, Switzerland, August 2-4, 2001

\title{
MEASUREMENT SCALES AND INTERVAL JUDGMENTS IN THE AHP
}

\section{Pekka Leskinen}

Finnish Forest Research Institute, Kannus Research Station, P.O. Box 44, FIN-69101 Kannus, Finland. Email: pekka.leskinen@metla.fi

Keywords: consistency, regression, statistical inference, uncertainty

Summary: Several numerical measurement scales for the elicitation of pairwise comparisons have been proposed in the AHP literature, but the evidence has been conflicting. At the same time, the choice of measurement scale have impacts on every AHP analysis through the estimated priorities and inconsistency. Besides the choice between alternative measurement scales, another problem is to give exact numerical values for the pairwise comparisons. This issue has been addressed through the use of interval judgments instead of accurate pairwise comparisons. In this presentation, the properties of alternative measurement scales and the probabilistic modelling of interval judgments are discussed in the framework of the regression analysis of pairwise comparisons data. The results supported the use of a geometric measurement scale. The probabilistic intervals enable more versatile analysis of uncertainties, and facilitate the interpretation of the differences between the measurement scales. 
